empty areas, formed on account of this lack of regeneration, becoine almost entirely occupied by the proliferated epithelial cells.

Considering that under optical examination these tissues appear empty, and that the opaque tissues (i.e., episcleral and scleral layers) which normally take part in the formation of the limbus region are absent in the pits area, it results that the lamellae of the cornea are visible through the proliferated epithelium which lies behind. Due to this fact the area corresponding to the pits is more transparent than normal.

Keeping pace with the sclerosis of both tissues and vessels situated between the nodules, the opacity corresponding to these is increased and reaches also the normally transparent part of the cornea. All these facts result in the appearance of a characteristic picture. This picture is made up of festoon-like lines of a whitish colour, which lines circumscribe clearly (with the exception of the edge of the cornea) the round-shaped greyish areas, which, on a superficial examination, suggest the impression of a pit, while in reality this is not the case.

Only an exceptionally small central depression can be found at their centre. In conclusion it must be said that the name "pit" used to indicate the lesions considered does not express the real morphology of these formations.

\title{
TWO CASES OF RETINAL DETACHMENT PRESENTING CERTAIN UNUSUAL FEATURES AFTER OPERATION BY SURFACE DIATHERMY
}

BY

\author{
H. B. Stallard
}

I.ONDON

IT occurred to me that certain features in the two cases of retinal detachment reported below might be of interest to some ophthalmic surgeons who are practising the surface diathermy technique of Larsson. Both cases were unsatisfactory during the immediate post-operative period of rest in bed in the appropriate position, the retina remaining detached, but later becoming completely replaced with full restoration of the visual field in each instance.

Case 1.-J. D., a boy, aged 13 years, was referred by his school doctor to the Moorfields Eye Hospital on account of defective left vision. He stated that this had been poor since some dirt had been thrown into his left eye four years ago. He had never worn glasses and was emmetropic in the right eye, the vision of which was $6 / 5$. 
The left eye proved subsequently to have $1.5 \mathrm{D}$. of hypermetropic astigmatism. The left vision was reduced to "hand movements" in the lower temporal part of his field. There was a retinal detachment occupying the position indicated in Fig. 1.

The macula was detached and presented a thin fenestrated appearance. At the ora serrata between " 4.30 and 6 o'clock" there

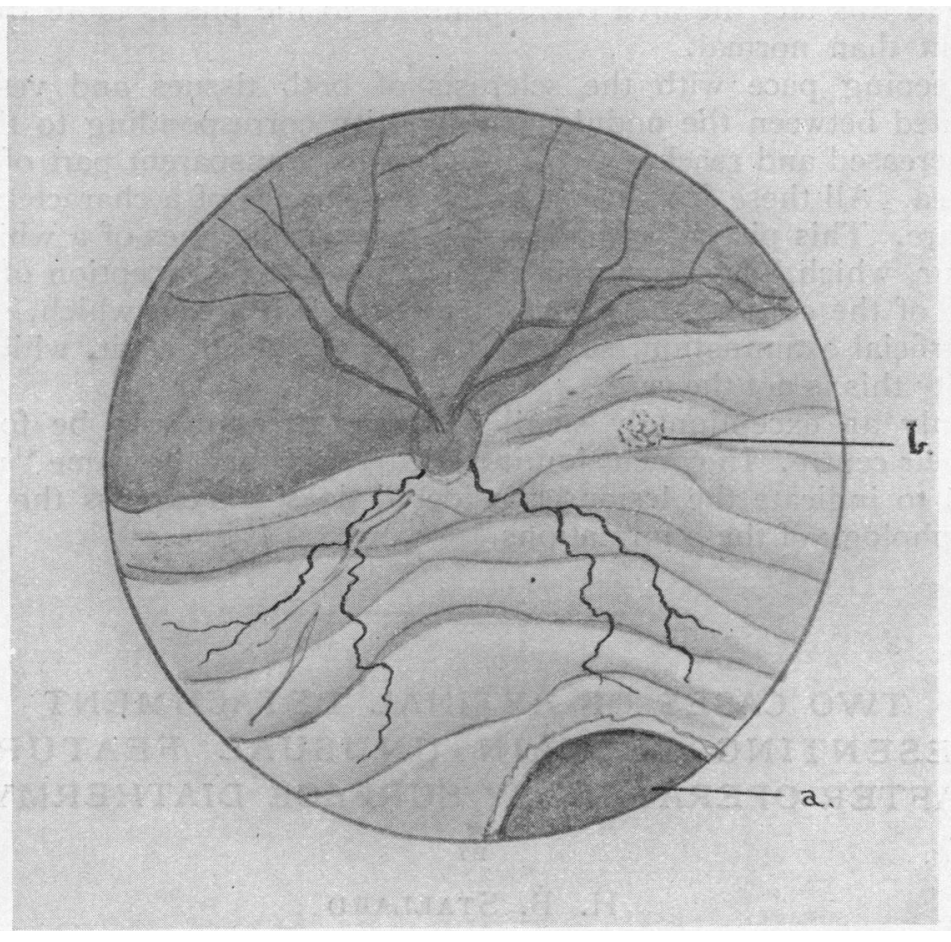

Fig. 1.

Diagram showing the site of the retinal detachment in Case 1.

a. Anterior retinal dialysis.

b. Detached macula presenting a thin fenestrated appearance.

was a large anterior dialysis with considerable retraction of the adjacent retina. Thin white lines, some running in the detached retina transversely from the optic disc above the macula and out towards the temporal side, and others along the course of the lower nasal branches of the central retinal vessels bore testimony to the $\omega$ long duration of the detachment. The detached area had an atrophic appearance. The vitreous was clear and of good quality as far as could be judged clinically. 
On February 3, 1934, an operation was embarked upon with some misgivings, which were realized early in the proceedings by the hopelessness of attempting any operative manoeuvre under a local anaesthetic and by the subsequent violence of the patient during induction under a general anaesthetic.

The operation was completed uneventfully, diathermy applications being made $3 \mathrm{~mm}$. apart over a band of sclera extending from " 4.30 to 6.30 o'clock" and at a depth between 8.5 and $15 \mathrm{~mm}$. behind the corneo-scleral junction. The inferior rectus muscle was retracted to gain access to that part of the sclera which it covered. A $1.5 \mathrm{~mm}$. trephine hole was made at the temporal edge of the inferior rectus muscle about $12 \mathrm{~mm}$. behind the limbus; inter-retinal fluid was evacuated and the conjunctival incision sutured.

Post-operative convalescence was marked by the patient's turbulence and restlessness. Reasoned argument, persuasion and attempts to exert restraint failing in turn we abandoned them and after a few days allowed him to move about as he wished.

On the 17th day after operation he was discharged from hospital. The anterior dialysis was occluded by white fluffy exudate and there was some inflammatory reaction in the choroid at the diathermy points, but the retina was detached and at this time I had the impression that the inter-retinal (sub-retinal) fluid was greater in volume than before the operation.

Three weeks after operation this state of affairs was unchanged, except that there was some scar-tissue formation in the vicinity of the anterior dialysis and at the diathermy points adjacent to this.

He re-attended the out-patient department three weeks later (six weeks after operation) with the retina completely replaced, the anterior dialysis closed and good choroido-retinal adhesions at the diathermy sites. The macula was replaced, his visual acuity was $6 / 36$, and the visual field full. The area of the retina which was formerly detached was clearly marked off from the remainder by having a faintly stippled and atrophic appearance. The white lines were still present, one running from the optic disc above the macula and outwards to the temporal side of the fundus was near the upper limit of the detachment, and another which followed closely the lower nasal branches of the central retinal vessels was unchanged in its position and relation to these vessels. The retina in the immediate vicinity of the macula was thinner than normal, but there were no gross degenerative changes. The remainder of the retina was healthy.

The retina has remained in place up to the date of writing this paper (September 24, 1934): the visual acuity is $6 / 24$ with +1.5 cyl., ax. $115^{\circ}$ and the visual field is full.

Case 2.-Mrs. S., aged 47 years, a patient referred to me through 
the kindness of Dr. D. Davies of Tunbridge Wells, had noticed a shadow in the right lower nasal quadrant of her visual field for $\vec{m}$ two months before I saw her on January 15, 1934. Dr. Davies hado noted a vitreous haemorrhage which on clearing revealed a retinalo detachment at the site shown in Fig. 2.

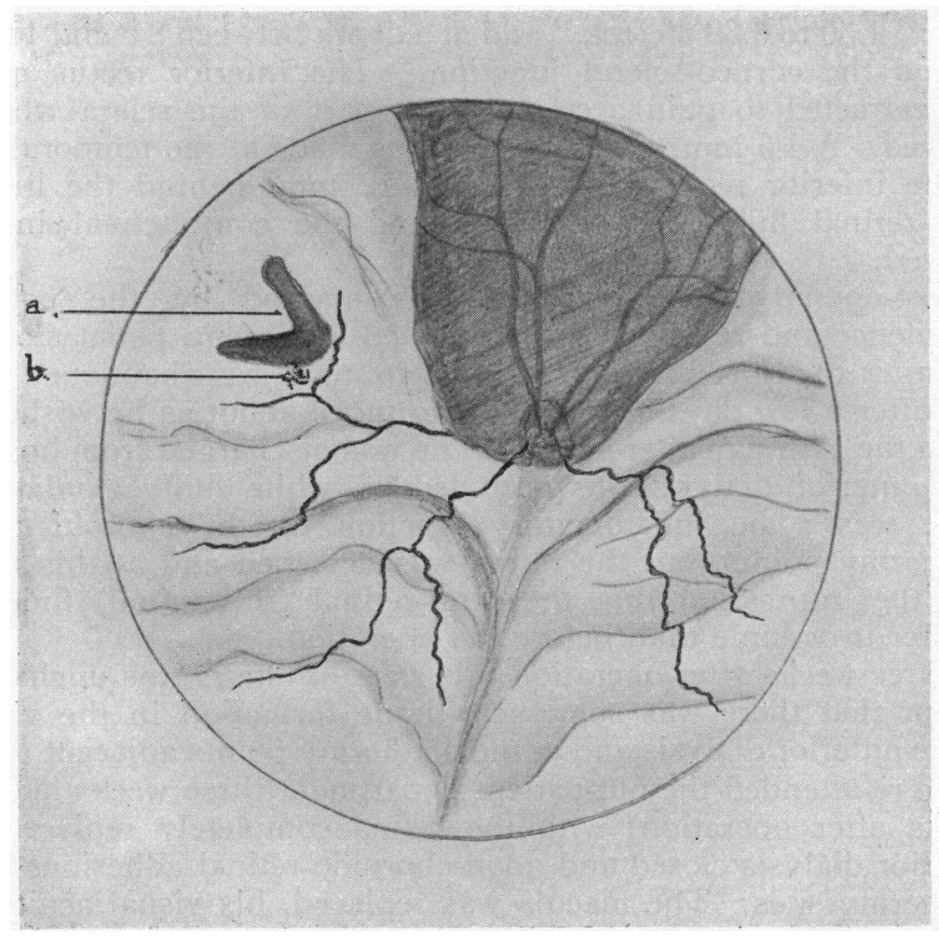

FIG 2

Diagram showing the site of the detached retina in Case 2.

a. Horse-shoe shaped retinal tear.

b. Small haemorrhage in the retina adjacent to the tear.

The macula was stripped off. In the " 10 o'clock" meridian and approximately 3 optic disc diameters from the ora serrata wase. a horse-shoe shaped retinal tear with its concavity facing upwardsu and outwards. A vessel ran in close proximity to this and theren was a small haemorrhage along the lower margin of the tear wheren a branch of this vessel evidently had been torn across. The right vision was reduced to hand movements.

Fine vitreous opacities and a generalized vitreous haze were्षे present in both eyes. There was no gross evidence of choroiditis in either eye. 
The left vision was $6 / 6$ and improved by +0.25 cyl., ax. $10^{\circ}$.

On February 3, 1934, an operation was performed. The sclera was exposed in the upper temporal quadrant of the right eye and surface diathermy applications made over an area from " 11 o'clock to 9 o'clock" and in depth 13 to $16 \mathrm{~mm}$. behind the limbus. A $1.5 \mathrm{~mm}$. trephine hole was made at the upper edge of the external rectus muscle about $14 \mathrm{~mm}$. bchind the limbus. Inter-retinal fluid was evacuated and the conjunctival wound sutured.

During the post-operative convalescence the retina became replaced in the upper temporal quadrant in the vicinity of the tear where satisfactory choroido-retinal adhesions were formed over the area treated by diathermy. Posterior to the tear the retina remained detached involving the macula and extending downwards affecting the lower half of the retina. The tear in the upper temporal quadrant was apparently closed and no other retinal hole was detected. The intra-ocular pressure was low. The inter-retinal fluid increased, and on the 14th day following operation after some deliberation I decided to make another row of surface diathermy applications posterior to the site of the tear in the upper temporal quadrant and to drain the inter-retinal fluid by making a trephine hole near the equator in the " 7 o'clock" meridian, having first surrounded this by a ring of diathermy applications before puncturing the choroid. This was done with some difficulty owing to the low intra-ocular pressure. The result was far from satisfactory. The eye remained very soft and the detachment, if anything, was more extensive. The choroido-retinal adhesions in the upper quadrant held fast and the tear was apparently closed. Two weeks after the second operation the detachment was a little shallower, but still present in the lower half of the globe involving the macula. The patient was allowed up on March 3 , and the condition of her eye was the same when she returned for examination on March 9. On April 20 much of the detachment was replaced. Two small areas of very shallow detachment were present, one adjacent to the macula on its temporal side and the other near the ora serrata in the " 6 o'clock" meridian. The retinal tear was still closed and good scarring was evident in its vicinity. On May 25 there was no sign of retinal detachment, the visual field was full and the vision $6 / 24$ (one letter) with $-1 \mathrm{sph} .+1.5 \mathrm{cyl}$. axis. $150^{\circ}$. Some dot-like lens opacities were noted and the vitreous showed a faint central haze.

On July 23 , the right vision had improved to $6 / 24$ (all the letters) with a correcting lens. Good choroido-retinal adhesions were noted in the upper temporal quadrant, the tear was sealed, the retina in place and the visual field full. There was no ophthalmoscopic evidence of gross disturbance at the macula. 


\section{Commentary}

These two cases have in common the facts that the eyes were almost emmetropic, and that although the immediate result of the surface diathermy was an apparent failure in each case the detached $\overline{\overline{\bar{s}}}$. retina and the field of vision were restored ultimately ; in case 1 , six weeks after operation, and in case 2 about three and a half months later.

The age of the patient, the duration and the site of the retinalo detachment and the position of the retinal tear differed in each case $\overrightarrow{\vec{\omega}}$ It is interesting to remark in passing that restoration of a retinalo detachment of long standing (probably four years) was possible. in a boy of 13 years of age whose powers of intelligent co-operation:and appreciation of the situation were negligible.

Such explanations as I offer for the somewhat dramatic course $\vec{\omega}_{\vec{\omega}}$ of events in these two cases are of a conjectural character. It iso probable that for some hours after the evacuation of the inter-retinal fluid through the scleral trephine hole there is an excessive pro-c duction of aqueous fluid some of which fails to be held in physicape combination with the vitreous gel and either drains away through the normal channels or seeps through the retinal tear, which at this stage is not closed by organized fibrous tissue or held down $8 y=$ adjacent choroido-retinal adhesions, and collects in the inter-retingl (sub-retinal) space. In some cases drainage through the scletailo trephine hole and choroid puncture may be slow or ineffective aftert the lapse of some hours owing to the occlusion of this exit channelo by inflammatory exudates. (However, it is a fact that leakage of intra-ocular fluid through a scleral trephine hole has been detected at a second operation three weeks after the first.)

The failure of the aqueous to form a stable physical combination with the vitreous gel, either through disease of that structure oro through chemical and physical alterations concerned in the main tenance of its hydrostatic equilibrium, causes a diminution of its. volume and hypotony, which affects the mechanical support of the retina.

The changes in the choroid after the application of diathermy are inflammatory in nature and it is possible in some cases for ano excessive amount of transudate to follow the hyperaemia and increased permeability of the chorio-capillaris. In many cases shallow layer of fluid is present in the inter-retinal space for an few days after operation and later becomes absorbed when the normal process of choroidal circulation is re-established and the्్ acute inflammatory phenomena are over. This dialysate is rich inc colloids and this will upset the osmotic pressure on either side of the detached retina, free aqueous which has failed to form a physicale combination with the vitreous gel passing through the retina ands increasing the volume of fluid in the inter-retinal space. 
In the cases under discussion there was no doubt, at any time, so far as could be judged by ophthalmoscopic examination, that the retinal tears were occluded by exudate. At first this occlusion would be of a cellular and fluid character and so would permit fluid to pass through it. Later, with the formation of fibrous tissue, such fluid filtration would be less free and eventually be cut off by the formation of firm adhesions between the retina and the choroid adjacent to the edges of the retinal tear.

It is probable that something of this nature occurred in these cases, the retinal tear becoming firmly sealed and the inter-retinal fluid gradually undergoing absorption as the hydrostatic equilibrium of the vitreous and the intra-ocular pressure became adjusted or re-established.

\title{
THE RESULT OF ORTHOPTIC TREATMENT IN DIVERGENT STRABISMUS
}

\section{BY}

\author{
Sheila Mayou
}

LON DON

THE purpose of this article is to consider the effects of orthoptic treatment on a series of cases of divergent strabismus of various types. As will be shown, orthoptic treatment plays an important part in every case, only a small number finally coming to operation. Of those operated on, all with the exception of one, had had previous training, which had developed fusion and some power of adduction before the operation, so that afterwards the desire for binocular vision enhanced and consolidated the success.

Out of 800 consecutive cases I find that 93 are cases of divergent strabismus. ${ }^{*}$ Of these 93 cases, 48 were emmetropic, 26 were hypermetropic, and 17 were myopic. The high percentage of these emmetropic cases occurring in this series is noteworthy, and I hope to show that these patients are the most favourable to treat.

It is well-known that the majority of convergent squints are hypermetropic and are often spontaneously cured by correcting the error of refraction. In my cases of divergent strabismus there is no error to correct, so that the improvement shown must be due to the exercises only, there being no other treatment which maly have been responsible for the cure.

\footnotetext{
* Constant concomitant (monocular or alternating) and occasional or periodic divergences are all included. Simple exophorias without demonstrable divergence are not included.
} 